\title{
Tagungsbericht
}

Tina Rotzal* und Nadine Walger

\section{HochSchulen in gemeinsamer Verantwortung: Welche Wert- und Qualitätsmaßstäbe vermittelt unser Bildungssystem?}

DOI 10.1515/iwp-2016-0008

Am 1. Dezember 2015 war die Johannes Gutenberg-Universität Mainz (JGU) Gastgeberin der Veranstaltungsreihe „U15 im Dialog“ der German U15. Über 80 Fachleute aus Politik, Schule und Wissenschaft kamen zusammen, um die Aspekte Integrität und Qualität im Kontext von Schule und Hochschule zu diskutieren. Die Veranstaltung, die in Kooperation mit der JGU und des Gutenberg Lehrkollegs (GLK) ausgerichtet wurde, war für alle Interessengruppen nicht nur inhaltlich, sondern auch organisatorisch sehr erfolgreich.

Die Grußworte überbrachten Georg Krausch (Präsident der JGU) und Michael Kißener (Stellvertretender Direktor des GLK). Beide waren sich einig, dass der universitären Lehre künftig mehr Beachtung zukommen müsse. So könne die Investition in eine gute Betreuung integres Verhalten von Studierenden und des wissenschaftlichen Nachwuchses fördern.

\section{Wissenschaftsethos im Niedergang?}

Keynote-Sprecher war Gerhard Fröhlich (Johannes Keppler-Universität Linz). Der Wissenschaftsforscher lieferte mit seinem Vortrag wichtige Denkanstöße zur Thematik. Eingangs benannte er Ursachen für Fehlverhalten. Es sei bedenklich, dass Öffentlichkeit und Medien dem „Schum-

1 Die German U15 ist ein strategischer Verbund von 15 forschungsstarken deutschen Universitäten zur Förderung von Wissenschaft, Forschung und Bildung.

*Kontaktpersonen: Tina Rotzal, M. A., Universitätsbibliothek Mainz, Jakob-Welder-Weg 6, 55128 Mainz, E-Mail: t.rotzal@ub.uni-mainz.de Nadine Walger, M. A., MA LIS, Deutsche Nationalbibliothek, Adickesallee 1, 60322 Frankfurt am Main, E-Mail: n.walger@dnb.de meln“ eine gewisse Sympathie entgegen brächten. Die Medien müssten sich ihrer Verantwortung gegenüber dem Hochschulsystem bewusster werden. Fröhlich kritisierte zudem das negative, allein auf Sensation ausgerichtete Wirken der Medien auf die Wissenschaft sowie die Präsentation von Wissenschaftserfolgen nach dem „HöherSchneller-Weiter-Prinzip“ aus der Sportwelt.

Er bemängelte des Weiteren die positive Einstellung der österreichischen Qualitätspresse zum Ghostwriting, das durch den Guttenberg-Effekt zugenommen habe. Schließlich tadelte Fröhlich die Kultur des „Nichts-Verpassen-Wollens" sowohl auf Seiten der Dozierenden als auch auf der der Studierenden und kritisierte, dass das Bluffen in unserer Gesellschaft zunehmend als Ikone gefeiert werde.

Fröhlich unterstrich, dass laut einer Studie wissenschaftliches Fehlverhalten nicht per se, wie oft angenommen, mit jüngeren Forschern einhergehe, sondern meist unter den sogenannten Mid Careers auftauche. Interessant sei, dass es meist der wissenschaftliche Nachwuchs gewesen sei, der zur Aufdeckung der großen Skandale beigetragen habe.

Wichtige Präventionsmaßnahmen seien neben einer Optimierung des Gutachtersystems, technologische Unterstützung und die Förderung von Kritik in der Wissenschaft. Letztlich trage auch Open Access dazu bei, Plagiate zu entdecken.

\section{Keine Kultur des Misstrauens schaffen}

Trotz aller Kritik - die Frage, ob sich das Wissenschaftsethos im Niedergang befände, verneinte Fröhlich.

Der letzte Programmpunkt des Vormittags war die von Mechthild Dreyer (Vizepräsidentin der JGU) moderierte Podiumsdiskussion zum Thema „Ist akademisches Fehlverhalten ein individuelles Phänomen? Zum Einfluss systemischer Faktoren auf akademische Integrität“. 
Einig war man sich, dass man keine Kultur des Misstrauens schaffen wolle - viele Plagiate gründeten eher in Unkenntnis, denn in Täuschungsabsicht. Die Werterziehung sollte in dieser Hinsicht bereits in der Schule beginnen und sich in der Hochschule fortsetzen. Das richtige Zitieren, Urheberschaft sowie Recht am eigenen Text und Bild werde ab der Unterstufe in der Schule thematisiert, so Christel Frey, Leiterin des Gymnasiums Nieder-Olm. Generell gebe es wenig Betrugsdelikte. Dagegen hatte Tanja Brühl, Vizepräsidentin der Goethe-Universität Frankfurt, den Eindruck, dass Studienanfänger zwar über gute Präsentationstechniken verfügen, dabei aber mehr auf die Ästhetik achteten, als auf die richtige Quellenangabe. Innerhalb der Universität sah sie die Problematik in der Betreuungssituation und plädierte für eine stärkere Rückmeldekultur: „Wenn ich mit Studierenden Fragestellungen entwickele, erkenne ich die eigenen Ideen, die eigene Leistung“. Man müsse deutlich machen, dass dies für die Lehre wichtig sei, und bei der Hausarbeit kommentieren, was erlaubt ist und was nicht. Ihr Amtskollege von der Technischen Universität Darmstadt, Ralph Bruder, pflichtete ihr bei, gab jedoch zu bedenken, dass die vermehrte Aufklärung auch zunehmend zu Verunsicherung geführt habe. Konsens war, dass eine gute Betreuungssituation mit weniger Fehlverhalten einhergehe, was allerdings mit den finanziellen und personellen Ressourcen sowie dem etablierten Betreuungssystem, auch in Hinblick auf Doktoranden, an den Universitäten aktuell nicht immer umzusetzen sei. „Qualität wächst unter solchen Bedingungen nicht unbedingt“, so Bruder. Brühl ergänzte, dass momentan Lehre und Studium ohne Drittmittel nicht angemessen gewährleistet werden könne, wies aber auch auf einen weiteren Faktor hin: „Wir brauchen Geld, aber wir brauchen auch ein Umdenken, was Prüfungsformen angeht." Je stärker standardisiert geprüft werde, desto eher gebe es Möglichkeiten zu plagiieren. Daher sprach sich Brühl für mehr kompetenzorientiertes Prüfen aus. Wichtig bei allen Punkten, ob Betreuungs- oder Prüfungsart, seien Absprachen zu Standards und Maßstäben. Denn wo liege die Zurechenbarkeit oder Schuld, wenn Leistungsstandards nicht klar seien, fragte Uwe Schmidt, Leiter des Zentrums für Qualitätssicherung und -entwicklung an der JGU. Individuellere, kompetenzorientierte Prüfungsformen, verbesserte Betreuung, frühes aber nicht verunsicherndes Ansprechen der Thematik - dies sind die Punkte, die bei der Prävention von Fehlverhalten wichtig sind. Und hierbei können Schulen und Hochschulen zusammen arbeiten: „Der Schlüssel ist, mehr gemeinsame Projekte durchzuführen“, so Frey, um Schüler, über einen Tag der offenen Tür hinausgehend, mit der Universität und dem wissenschaftlichen Arbeiten vertraut zu machen.

\section{Werteerziehung und -vermittlung in Schule und Lehrerausbildung}

Nach der Mittagspause wurde die Veranstaltung in zwei parallelen Sektionen fortgeführt. Sektion 1 fokussierte auf das Thema Werteerziehung und -vermittlung in der Schule und in der Lehrerausbildung.

Der „Werterziehung als Qualitätsdimension von Schule und Unterricht“ widmete sich Jürgen Rekus (Universität Karlsruhe). Bildung würde heute nicht mehr dem einst anthropologischen Sinn gerecht, sondern sei ein Wirtschaftsgut, das sich dementsprechend lohnen müsse. Bildung würde oftmals auf den Begriff Ausbildung herunter gebrochen. Es werde zunehmend nach Standards und nach deren Messbarkeit gefragt.

Das Interessante sei aber, dass das, was sich nicht messen lässt, die Einmaligkeit eines Menschen ausmache. Für Rekus besteht Bildung aus der Trias von Wissen, Werten und Entscheiden. Lehrkräfte müssten, und dies sei die große Herausforderung, Schüler demnach zu Werturteilsfähigkeit anleiten, damit sie schließlich handlungsfähig werden. In einer Gesellschaft, die keine Werte mehr vorgeben kann, sei es unabdinglich, die Vielfalt der Werte überschauen zu können und sich selbst zu positionieren. Angehende Lehrkräfte seien darin zu schulen, Unterrichtsinhalte selbst pädagogisch nach folgenden Kriterien zu werten: Zweckhaftigkeit, Conditio Humana, Sittlichkeit und Sinn.

Ursula Jünger (Friedrich-Ebert-Gymnasium Bonn, Zentrum für schulpraktische Ausbildung Bonn) berichtete, dass das Thema des Fehlverhaltens, in der Schule unter dem Begriff „Schummeln“ gefasst, zwar in Schule und Lehrerausbildung wichtig sei und angesprochen werde, von Referendaren und Schülern aber häufig eher beiläufig wahrgenommen werde. Voraussetzung für die Vermittlung von Normen und ethischen Werten sei jedoch die reflektierende Auseinandersetzung mit diesen. Respekt im Umgang miteinander gelte sowohl bei Schülern als auch Referendaren als „bei Weitem bedeutsamster Wert“. Hierin sah Jünger eine gute Ausgangssituation, um das Thema der (akademischen) Integrität auf einer anderen Ebene anzugehen und zu zeigen, dass Schummeln und Plagiieren auch eine moralische Dimension haben. In den entsprechenden Materialien für die Lehrerausbildung, z.B. zum ,materialgestützten Schreiben', werde aber eher auf juristische Gründe verwiesen. Grundsätzlich sei es unerlässlich, dass die Werteerziehung mit den Inhalten der Lehrerausbildung vernetzt werde. Es sei nicht zielführend, wenn diese Werte und Standards nur mit einzelnen Individuen und Institutionen verbunden werden. Vielmehr müsse 
Redlichkeit und eigenständige Arbeit als eigener WerteStandard angenommen werden. Diese Haltung könne im Referendariat und auch beim Schulunterricht durch die Diskussion von Fallbeispielen und Reflexion des eigenen Verhaltens und der eigenen Maßstäbe befördert werden dies sollte wiederholt und häufig passieren.

\section{Prävention von wissenschaftlichem Fehlverhalten}

Sektion 2 beschäftigte sich mit Möglichkeiten der Prävention von wissenschaftlichem Fehlverhalten.

Dominik Schuh und Nicole Walger (beide Universitätsbibliothek (UB) Mainz) stellten das an der UB Mainz angesiedelte Projekt „Akademische Integrität“2 vor. Sie konstatierten, dass sich Fehlverhalten im akademischen Alltag nicht gänzlich vermeiden ließe, dies aber das Vertrauen in die Wissenschaft sowie innerhalb dieser gefährde. Sie diskutierten vier Handlungsprämissen, von denen zwei die Botschaft enthielten, dass akademisches Fehlverhalten zu weiten Teilen unbeabsichtigt auftrete. Diese Auffassung bilde die Grundlage des Projekts. Das optimistische Menschenbild, das der Vortrag damit nach außen trägt, führt zu der These, dass der Wille zum Guten mit der Möglichkeit seiner Umsetzung zusammentreffen müsse. Dieser Wille ließe sich jedoch nur bedingt beeinflussen. Eine besondere Herausforderung bei der Vermittlung der Regeln und Werte der guten wissenschaftlichen Praxis seien die Grauzonen der Regelungen, die nicht abschließend zu klären seien. Ein wichtiger Schritt im Kontext der Grauzonenklärung sei einerseits die Sichtbarmachung der Regeln im universitären Raum, zum anderen eigne sich zu diesem Zweck auch die Bearbeitung und Diskussion von Fallbeispielen.

Schuh und Walger thematisierten zudem die Frage, wie im Kontext der akademischen Integrität universitätsübergreifend agiert werden könne und welche Standards dafür zu schaffen seien. Neben Werkzeugen zur Vermittlung von wissenschaftlichen Arbeitstechniken, wiesen die Referenten auf E-Learning-Materialien und Veranstaltungen des Projekts hin. Generell betonten beide jedoch, dass reine Online-Angebote in einem sensiblen Kontext wie diesem kein Ersatz für den persönlichen Austausch und Kontakt mit Menschen seien. Die abschließende Diskussi-

2 Das Projekt ist Teil des vom BMBF geförderten Projekts „Lehren, Organisieren, Beraten (LOB) - Gelingensbedingungen von Bologna“ https://www.lob.uni-mainz.de/ [08.12.2015]. on zeigte weitere Handlungsfelder auf und man war sich einig, dass Projekte dieser Art immer eine gewisse Pionierarbeit leisten.

Eleonora Kohl (Pädagogische Hochschule (PH) Freiburg) berichtete von dem Projekt „Freiwillige Plagiatskontrolle (FPK)“ der PH sowie dem Projekt „ReFAIRenz“, an dem die PH Freiburg, die Universität Konstanz und die HTWG Konstanz beteiligt sind. Beiden Projekten liege zunächst der Forschungsgedanke zugrunde, verschiedene Arten von Plagiaten/Fehlern sowohl fach- als auch hochschulspezifisch $\mathrm{zu}$ identifizieren, um hochschuldidaktische Maßnahmen besser darauf abstimmen und entwickeln zu können. Die kooperierenden Hochschulen arbeiten hier mit dem Begriff des ,intertextuellen Fehlers', der darauf Bezug nimmt, dass in jedem Text andere Texte vorkommen und Verwendung finden: „Nicht jede falsch oder ungenügend gekennzeichnete Textstelle ist ein (absichtliches) Plagiat“, so Kohl. So seien bisher auf Grundlage eines Korpus von schriftlichen Arbeiten aus verschiedenen Qualifizierungsstufen, Fächern und Hochschultypen, hundert intertextuelle Fehlertypen identifiziert worden. Die ,inkorrekte Intertextualität‘ biete sich im Forschungskontext als wertungsneutrale Alternative an, mit der ein Generalverdacht vermieden werde.

Bei der FPK haben Studierende die Möglichkeit, ihre schriftlichen Arbeiten einzureichen und mit Hilfe einer Plagiatsdetektionssoftware prüfen zu lassen. Sie erhalten eine Rückmeldung, die Bezug auf die Fehlerarten nimmt, die aufgetreten sind, und Verbesserungsvorschläge gibt. So hätten Studierende die Chance, aus Fehlern und Unbedachtheiten zu lernen, bevor es ,ernst' werde. In 90 Prozent aller Arbeiten von Studierenden der ersten drei Semester traten Zitierfehler oder fehlende/falsche Belege auf - allerdings wurden davon nur drei Prozent als (Teil-) Plagiate eingestuft. Dies weist eher auf einen Kompetenzmangel im Umgang mit Texten und Ideen andere, denn auf eine Kultur des bewussten Täuschens hin.

\section{Dialog mit der Lehre}

Im Schlusswort wies Mechthild Dreyer darauf hin, dass der Dialog mit der Lehre noch am Anfang stehe. Die Veranstaltung hätte jedoch die Basis dafür geschaffen, dass verschiedene Akteure miteinander ins Gespräch kamen und Gespräche in verschiedene Richtungen weiter fortgesetzt werden können.

Abschließend lässt sich folgendes Fazit ziehen: Akademische Integrität ist eine Gemeinschaftsaufgabe und erfolgreiche und innovative universitäre Lehre ist in diesem Kontext nur möglich, wenn Schulen und Hochschulen 
kooperativ und intensiv zusammenarbeiten. In gemeinsamen Projekten können beide Seiten sowohl voneinander als auch miteinander lernen. Und gerade wenn es um die Vermittlung von Wert- und Qualitätsmaßstäben geht, kann auch der Blick über den deutschen Tellerrand hinaus neue Impulse liefern. Der Vermittlung von Medien- und Informationskompetenz kommt besonders an der Schnittstelle von Schule und Hochschule eine wichtige Rolle zu.

Die Tagungsbroschüre, die Präsentationsfolien sowie bearbeitete Aufzeichnungen der Vorträge stehen Interessierten auf der U15-Website zur Verfügung ${ }^{3}$.

Deskriptoren: Tagung, Schule, Hochschule, Wissenschaftliches Arbeiten, Zitat, Qualität, Akademische Integrität, Informationskompetenz, Medienkompetenz

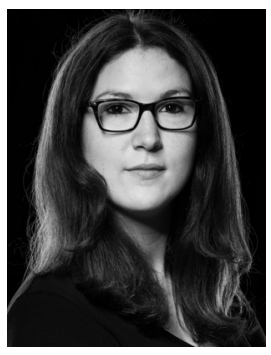

\section{Tina Rotzal, M. A.}

Universitätsbibliothek Mainz

Jakob-Welder-Weg 6

55128 Mainz

t.rotzal@ub.uni-mainz.de

Foto: Thomas Hartmann

Tina Rotzal, M. A. ist Mitarbeiterin des Projekts „Akademische Integrität" an der Universitätsbibliothek Mainz. Sie studierte Germanistik, Anglistik und Kulturanthropologie in Mainz und arbeitet an einer Dissertation zur Entwicklung der Großschreibung im Deutschen. Interessenschwerpunkte: Prävention von Fehlverhalten an Hochschulen und Schulen, Vermittlung von Schreib- und Informationskompetenzen.

3 Vgl. dazu: Homepage German U15 http://www.german-u15.de/in dex.html [08.12.2015].

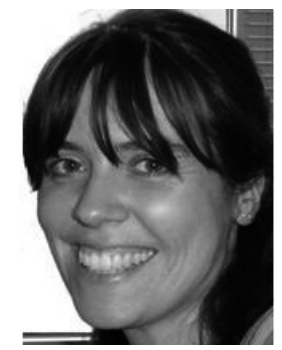

Nadine Walger, M. A., MA LIS

Deutsche Nationalbibliothek

Adickesallee 1

60322 Frankfurt am Main

n.walger@dnb.de

Nadine Walger, M. A., MA LIS ist Mitarbeiterin der Deutschen Nationalbibliothek in Frankfurt am Main. Sie studierte Anglistik und Germanistik in Frankfurt am Main sowie Bibliotheks- und Informationswissenschaften in Köln. Interessenschwerpunkte: Vermittlung von Medien- und Informationskompetenz an Schulen und Hochschulen, wissenschaftliches Publizieren sowie ethische und soziologische Fragen des Bibliotheks- und Informationswesens. 